\title{
Effect of filler amount on thermoelastic properties of poly(dimethylsiloxane) networks
}

\author{
Mustafa M. Demir ${ }^{\mathrm{a}, 1}$, Yusuf Z. Menceloglu ${ }^{\mathrm{a}}$, Burak Erman ${ }^{\mathrm{b}, *}$ \\ ${ }^{a}$ Faculty of Engineering and Sciences, Sabanci University, Tuzla, 34956 Istanbul, Turkey \\ ${ }^{\mathrm{b}}$ Department of Chemical and Biological Engineering, Koc University, Rumelifeneri, Sariyer, 34450 Istanbul, Turkey
}

Available online 8 March 2005

\begin{abstract}
End-linked poly(dimethylsiloxane) (PDMS) networks were prepared in the presence of fumed silica particles with hydroxyl groups at their surfaces. The silica particles were introduced into the polymer solution prior to end-linking. Hydroxyl ended PDMS chains were end-linked via the tetra functional crosslinker, tetraethoxysilane. The filler content varied in the range $0-5 \mathrm{wt} \%$. Atomic Force Microscopy was used to image and characterize the silica particles. Swelling, stress-strain and thermoelasticity experiments were performed. The temperature coefficient and the energetic part of the force in uniaxial extension are found to increase with increasing silica amount. This observation is ascribed to effects contributed possibly by the adsorption layer around the silica particles.
\end{abstract}

(C) 2005 Elsevier Ltd. All rights reserved.

Keywords: Poly(dimethylsiloxane); Thermoelasticity; Fumed silica

\section{Introduction}

Poly(dimethylsiloxane) (PDMS) is a well-known member of the siloxane family, which is heavily used in the industry. The importance of PDMS comes from its good electrical properties, optical clarity, weather resistance and very low glass transition temperature, $T_{\mathrm{g}}\left(\sim-120^{\circ} \mathrm{C}\right)$. Its elastic properties cover a large range of temperatures. However, its mechanical properties are very poor. Additives, particularly silica, are used to improve its mechanical properties and also to protect the optical clarity. There are very few applications of the PDMS elastomers used in the unfilled state. Filler morphology, which is described as particle size and structure, plays a significant role on the degree of elastomer reinforcement [1]. Good dispersion of particles in the elastomer is crucial for achieving qualities. Yuan and Mark reported that precipitation of the silica particles in situ provides better dispersion of the filler and enhances reinforcement [2]. The statistical properties of

\footnotetext{
* Corresponding author. Tel.: +90 212338 1704; fax: +90 212338 1548.

E-mail address: berman@ku.edu.tr (B. Erman).

${ }^{1}$ Current address: Max Planck Institute for Polymer Research, Ackermannweg 10, D-55021 Postfach, 3148 Mainz, Germany.
}

0032-3861/\$ - see front matter (C) 2005 Elsevier Ltd. All rights reserved. doi:10.1016/j.polymer.2005.02.053
PDMS chains are markedly different than other chains used in the commonly used elastomers. Since, the $\mathrm{Si}-\mathrm{O}$ bond is rotationally flexible, PDMS shows an unusually high degree of chain flexibility. Alternating bond angles in the PDMS chain and the ionic nature of the $\mathrm{Si}-\mathrm{O}$ bond leads to highly variable conformational behavior.

The reinforcement of PDMS elastomers by fillers has been the subject of numerous studies [2,3]. Earlier work on filled PDMS concentrated on the effects of fillers on macroscopic properties several examples to which are given in $[4,5]$. The role of molecular structure at the polymer-filler interface on the filler effect is considered only in recent years. Notably, Litvinov et al. studied, by NMR, the structure and behavior of grafted PDMS chains on silica and found that the layer consists of immobilized chain segments at the PDMS-silica interface and mobile chain portions outside the interface [6]. Chains at the interface exhibited substantial decrease in heat capacity at the glass transition temperature. Some of the PDMS chain units were immobilized as a result of physical adsorption at the silica surface, the amount being proportional to the number of silanol groups at the silica surface. Gussoni et al. also used NMR spectroscopy to investigate silica filled PDMS rubbers, and observed the decrease of segmental mobility of the PDMS chains in the vicinity of the filler surface [7]. Berriot et al. employed ${ }^{1} \mathrm{H}$ NMR experiments on filled rubbers and observed a layer of immobilized segments 
at the particle surface [8]. In the case of chains chemically grafted to the silica surface, Berriot et al. observed immobile layers, which they described as a glassy shell around the filler surface. Cosgrove et al. used differential scanning calorimetry and NMR measurements, and showed that the reduction in the mobility of the PDMS chains corresponded to a shift in the glass transition to higher temperatures [9]. These shifts are relatively small. Nevertheless, they indicate the presence of a gradient of segmental mobility in going from the rubber towards the silica surface.

For an end-linked PDMS network filled with $1 \%$ by weight silica particles of about $75 \mathrm{~nm}$ diameter, the average spacing between the surfaces of two neighboring silica particles, assuming good dispersion, is in the order of $75 \mathrm{~nm}$ (see Appendix B for an estimate of this value). For 5\% filler, this distance falls to $50 \mathrm{~nm}$. The average end-to-end distance of a PDMS chain of 53,000 molecular weight is about $10.6 \mathrm{~nm}$. Therefore, the distance between two neighboring silica particles is spanned by 5-7 network chains. Each extremity of the path of 5-7 chains is embedded in a layer of diminished mobility. The thickness of the layer is not negligible. One would expect strong thermoelastic effects arising from the presence of mobility gradients in the elastomer, which may accurately be determined by measuring the energetic contribution of the restoring force in a thermoelastic experiment. The specific aim of the present work is to study the effect of fillers on the thermoelasticity of silica filled PDMS networks.

Thermoelasticity of filled cross-linked PDMS was studied earlier by Galanti and Sperling who obtained a strong increase in the energetic contribution to the elastic force with increasing filler content [10]. Our results qualitatively agree with their results, with the quantitative difference that the energetic contribution in our measurements increases more strongly than theirs with filler amount. A precise quantitative characterization of the filler effect in PDMS networks is important due to their use in technological applications. Sperling's results and ours thus set two different levels of thermoelasticity which hopefully will further be investigated by others. Galanti and Sperling interpreted their results in terms of a semi-quantitative equation of state and attributed the increase in energetic contributions to filler-rubber interface. Their interpretation is now validated by the precise NMR observations, which we discuss further in the Section 4.

\section{Experiments and theory}

Untreated fumed silica, provided from CAB-O-SIL was used. The specifications and typical properties of the filler are as follows: The particle size of the silica is $14 \mathrm{~nm}$ and surface area is $200 \pm 25 \mathrm{~m}^{2} / \mathrm{g}$. For surface chemistry, hydroxyl groups cover approximately $45 \%$ of the surface of the silica making the surface hydrophilic. Elastic modulus and thermal expansion coefficient of bulk amorphous silica are $72.4 \times 10^{9} \mathrm{~m}^{-1} \mathrm{~kg} \mathrm{~s}^{-2}$ and $0.5 \times$ $10^{-6} /{ }^{\circ} \mathrm{C}$ respectively [11].

\subsection{Network synthesis}

The hydroxyl terminated PDMS was end-linked into a network by reacting with an excess amount of the crosslinking agent, tetraethoxysilane (TEOS). The molecular weight of the PDMS is $53 \mathrm{kDa}\left(n_{\mathrm{w}}=675\right)$, where $n_{\mathrm{w}}$ is the weight average degree of polymerization. Polydispersity was close to 1.62 . The end-linking reaction was performed in solution to facilitate the mixing process and the samples were sonicated to obtain better dispersion of silica particles. The hydroxyl ended PDMS was dissolved in toluene. The desired amount (unfilled, 1, 3, 5 wt \%) of silica particles was added to the solution. The mixtures were stirred and later sonicated for $2 \mathrm{~min}$ to obtain a homogeneous dispersion. The favorable dispersion effects of sonication were observed by comparing with the atomic force microscope images of samples prepared without sonication. Six hundred microlitre TEOS and the catalyst, Tin(II) 2-ethylhexanoate, were added to the dispersion while stirring. The crosslinking reactions were carried out in a Teflon mold at room temperature for $24 \mathrm{~h}$ and at $80{ }^{\circ} \mathrm{C}$ overnight. The thickness of the resulting film was on the order of $1.5-2 \mathrm{~mm}$. The amount of solvent during the end-linking process is not constant due to evaporation of toluene. An average value of 0.50 is estimated for the volume fraction of polymer during the end-linking process. Higher silica concentration $(>5 \% \mathrm{wt}$ ) in PDMS films could not be prepared due to silica agglomeration. This issue will be discussed further in the Section 3.

\subsection{AFM imaging}

Tapping mode in air Atomic Force Microscopy (AFM) (Nanoscope IIIa, Digital Instruments) imaging was carried out with oxide sharpened Si tips. Sample surfaces were scanned without any surface preparation. AFM images are recorded in the height and phase modes. Dimensions of the silica particles were measured by the image analysis options of the Nanoscope software that provides the density and mean diameter of aggregates.

\subsection{Sol-gel analysis and swelling experiments}

Sol-gel analysis was performed to determine the crosslinking degree in two different solvents, toluene and benzene. A small piece of the unfilled end-linked PDMS film was placed in a $30 \mathrm{ml}$ of reagent grade toluene or benzene. The sample was allowed to remain in the solvent for 2 days at room temperature. The swollen gel was taken from the solvent and dried in a vacuum oven at room temperature. The sol fraction in the end-linked sample was calculated from 
$\%$ sol fraction $=\left[\left(\left(m_{0}-m_{\mathrm{e}}\right) / m_{0}\right) \times 100\right]$

where $m_{\mathrm{e}}$ is the dry mass of the extracted sample and $m_{0}$ is the initial mass of the sample.

Swelling experiments of the end-linked PDMS samples were carried out in the same two solvents. The polymer films were allowed to remain in the solvent for $24 \mathrm{~h}$ at room temperature and the dimensions of the swollen films were measured as soon as they were taken out of the solvent. Swelling ratios $q_{\mathrm{v}}$ of networks

$q_{\mathrm{v}}=$ volume of swollen network/volume of dry network

at equilibrium were determined.

\subsection{Thermoelasticity measurements}

The dimensions of the samples on which the thermoelasticity measurements were performed were $40 \times 2 \times$ $2.5 \mathrm{~mm}^{3}$. The ends of the sample were mounted between two metal clamps. The lower clamp was fixed and the upper one was connected to a movable force transducer. A short condenser was designed as a temperature chamber. Hot silicone oil was circulated with a temperature-regulated pump (Haake DC 30). Two small points were marked on the sample with a permanent marker. A JVC TK-C 1381 camera attached to an Olympus SZ-STUZ optical microscope was used to image the points. Displacements of the marked points were followed via an image analysis program by which the distance between the centers of points was measured in pixels. A sensitive force transducer (TSD125F, Biopac systems, Inc.) was employed for force measurement. The length between the two centers was kept constant with the help of a tension adjuster (HDW100A) that provides micron-scale control. The stress on the film was read out from the multimeter (Agilent 34401A) in terms of potential difference in volts. A schematic view of the experimental apparatus is presented in Fig. 1. The transducer was calibrated by relating the potential difference into a force unit, by applying a set of dead weights on the transducer. Potential differences corresponding to the masses were recorded and plotted as a function of force. A linear calibration curve was obtained with a good regression value $\left(R^{2}=0.968\right)$.

The sample was loaded and heated in a glass chamber. $120 \pm 1{ }^{\circ} \mathrm{C}$ was the highest temperature employed, and $16 \mathrm{~h}$ were allowed for chain relaxation. Next, a stepwise cooling was performed on the system at $20{ }^{\circ} \mathrm{C}$ intervals down to room temperature. A sufficient time, more than $2 \mathrm{~h}$, was allowed to bring the system to equilibrium between two successive measurements. Force data were recorded by the help of a HP-GPIB data acquisition card. The crosssectional area of the undeformed sample was determined by measuring the thickness and width of each sample with a micrometer with a precision $100 \mu \mathrm{m}$.

\subsection{Mechanical measurements}

Stress-strain measurements were obtained at room temperature in uniaxial extension and along the direction of increasing elongations. Stress, $\sigma$, is calculated as $\sigma=f / A_{0}$; where, $f$ and $A_{0}$ are the measured force and the undeformed cross-sectional area, respectively.

\subsection{Thermoelasticity theory}

The elastic force $(f)$ has two components, an internal energy component $\left(f_{\mathrm{e}}\right)$ and an entropic component $\left(f_{\mathrm{s}}\right)$. The latter results from the decrease in conformational entropy due to the deformation of the polymer chains to a less probable state. The end-linked PDMS samples are stretched to a fixed length $(L)$ and the force $(f)$ is measured as a function of temperature at constant pressure $(p)$. The energetic origin of the applied force is calculated according to [4]

$f_{\mathrm{e}} / f=-T[\partial \ln (f / T) / \partial T]_{L, p}-\left(\beta T /\left(a^{3}-1\right)\right)$

Here, $\beta$ is the thermal expansion coefficient taken as $15 \times 10^{-6} \mathrm{~K}^{-1}$ for unfilled samples [12]. $\beta$ of filled elastomers were calculated according to the following formula: wt $\%$ silica $\times \beta$ of silica $+(1-\mathrm{wt} \%$ silica $) \times \beta$ of pdms.

$\alpha$ is the extension ratio $L / L_{0}, f$ is the force and $T$ is the absolute temperature. Dividing through by $T$, and using the relation $f_{\mathrm{e}} / f=T \mathrm{~d} \ln \left\langle r^{2}\right\rangle_{0} / \mathrm{d} T$, we get

$\mathrm{d} \ln \left\langle r^{2}\right\rangle_{0} / \mathrm{d} T=-[\partial \ln (f / T) / \partial T]_{L, p}-\left(\beta /\left(\alpha^{3}-1\right)\right)$

Eq. (4) relates microscopic information carried in the left hand side to macroscopic measurements given by the right hand side. The derivation of this equation is described in the original Refs. [13-15].

The unperturbed end-to-end distance, $\left\langle r^{2}\right\rangle_{0}$, can be estimated from $\left\langle r^{2}\right\rangle_{0}=C_{M} n l^{2} . C_{\mathrm{M}}$ is the characteristic ratio at finite molecular weight, which was estimated by Flory for PDMS [16] using the rotational isomeric state theory to be in the range 5.85-7.60. $n$ is the number of repeat units (675) and $l=1.67 \AA$ is the $\mathrm{Si}-\mathrm{O}$ bond length. Thus, the end-to-end distance is estimated as $10.6 \mathrm{~nm}$.

\section{Results}

\subsection{AFM imaging and particle size}

The morphology of silica filled PDMS is presented in Fig. 2. The background of the image is the PDMS matrix and the bright objects are the silica fillers. Aggregate density and mean aggregate diameter was measured for each sample. Results of measurements are given in Table 1. Dispersion of the silica particles becomes relatively poor as silica concentration increases. When the silica concentration 


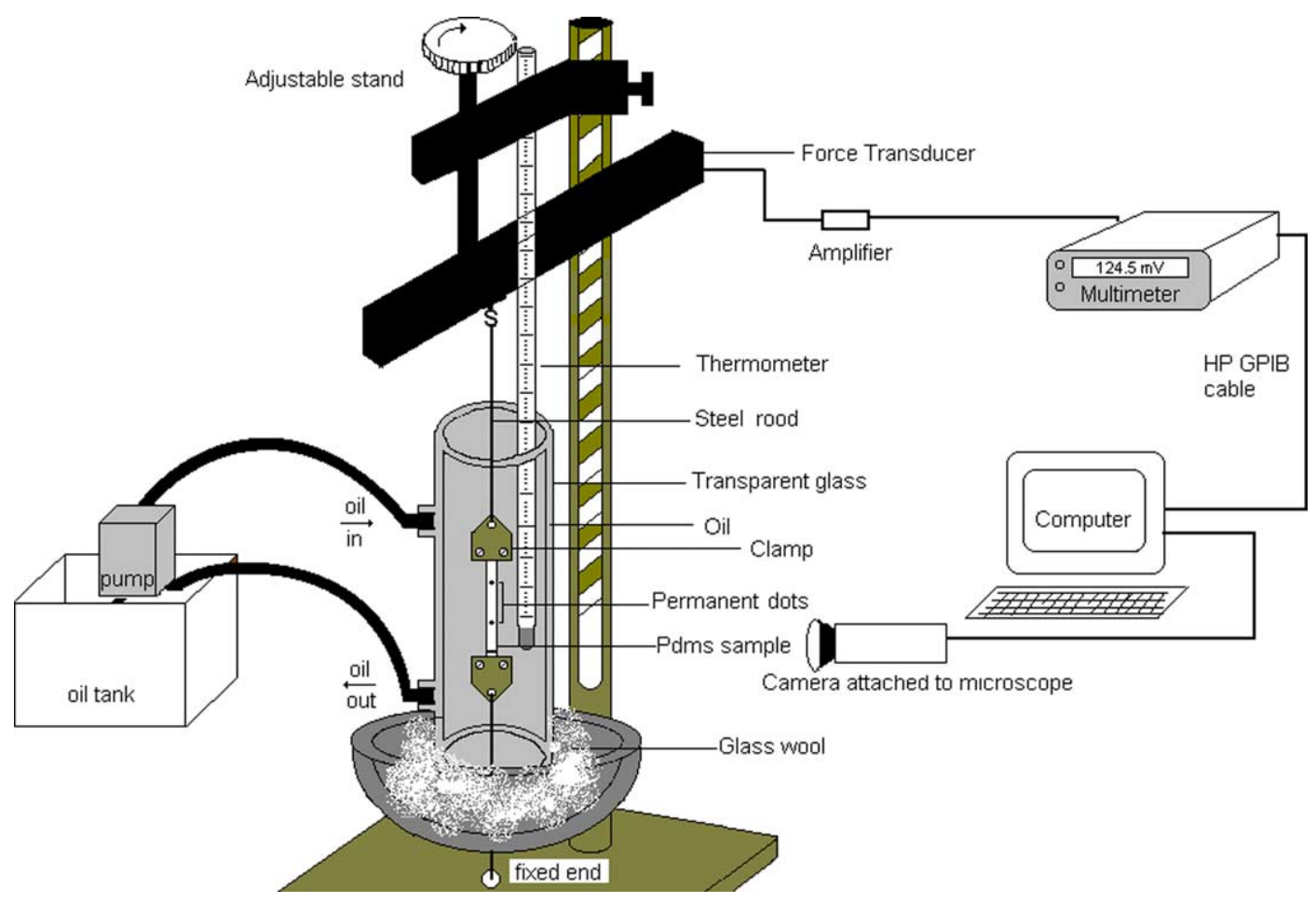

Fig. 1. Schematic view of experimental apparatus.

is higher, the number of silica particles per unit area becomes markedly less and mean particle diameter increases. The primary silica particles have $14 \mathrm{~nm}$ diameters, but they tend to aggregate, and to form larger agglomerates to reduce the total surface energy. Fig. 2(a) is for the $1 \%$ silica sample. It shows that the filler is uniformly distributed in the PDMS matrix. The tendency of the filler particles to agglomerate is enhanced as the silica concentration increases as shown in Fig. 2(b) for the 3\% and Fig. 2(c) for the $5 \%$ silica samples.

Fillers with smaller particle size, i.e. higher effective surface area are favored for rubber reinforcement. Better reinforcement is achieved by increasing the effective surface area, which enhances the contact area between the polymer and filler. At higher concentrations, the particles become larger and form both aggregates and agglomerates. Thus, it is difficult to control the particle size and degree of dispersion due to agglomeration.

\subsection{Swelling measurements and sol-gel analysis}

Results of equilibrium swelling measurements are presented in Table 2. The second, third and fourth columns give the elastic moduli of networks from swelling in toluene, benzene, and from mechanical measurements, respectively. The last three columns give the molecular weight of network chains, $M_{\mathrm{c}}$ (obtained by equations of Appendix A). The amount of material extracted is small for swelling in both benzene and toluene, varying in the range $2-4.2 \%$ by weight. Swelling degrees of the end-linked
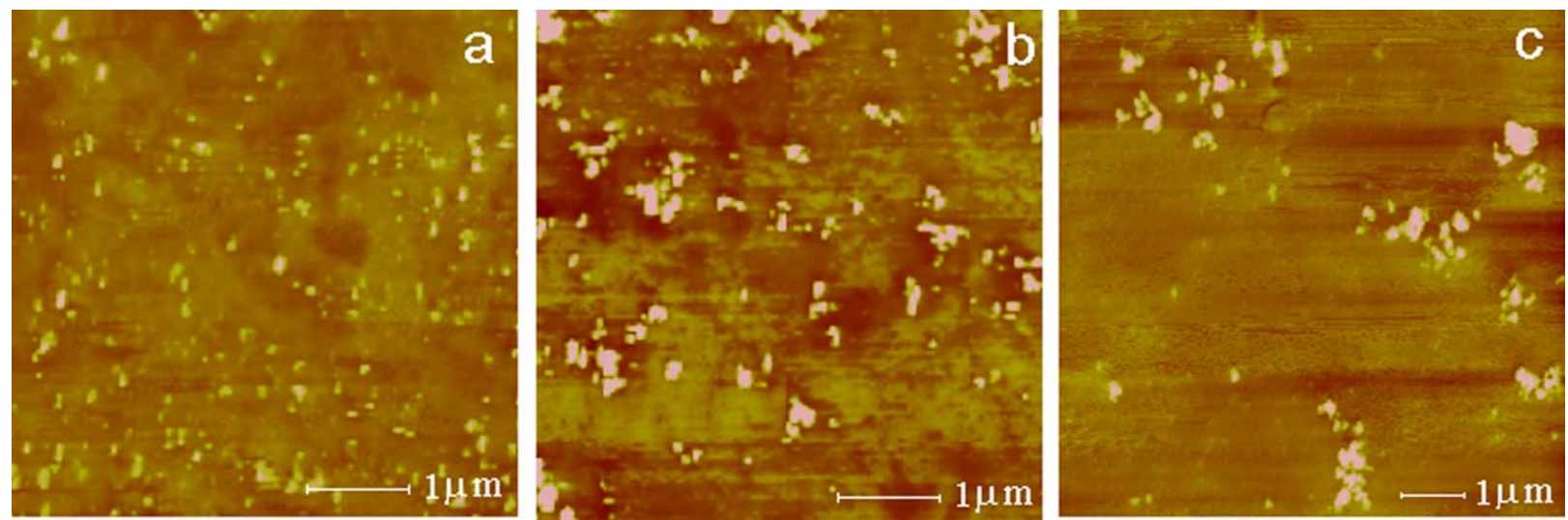

Fig. 2. AFM tapping mode phase image of silica aggregate in PDMS matrix, (a) $1 \% \mathrm{wt}$; (b) $3 \% \mathrm{wt}$; (c) $5 \%$ wt silica. 
Table 1

Density and diameter of silica aggregates

\begin{tabular}{llc}
\hline Silica conc. (\%wt) & Aggregate density/ $\mu \mathrm{m}^{2}$ & $\begin{array}{l}\text { Mean aggregate } \\
\text { diameter }(\mathrm{nm})\end{array}$ \\
\hline 1 & 7.83 & 76 \\
3 & 3.91 & 101 \\
5 & 0.62 & 201 \\
\hline
\end{tabular}

PDMS in the two solvents are presented in Fig. 3 as a function of the amount of silica filler.

\subsection{Elastomeric force and temperature coefficient}

$\ln (f / T)$ vs. $T$ was plotted to obtain the slope $\partial \ln (f / T) / \partial T$ for each sample. Fig. 4 is given as a representative plot among the other samples (unfilled, 1\%, 3\% wt silica). All samples exhibit similar behavior, $\ln (f / T)$ decreases linearly as temperature increases. The slopes of the curves are critical in determining the $f_{\mathrm{e}} / f$ value, Eq. (3).

Fig. 5 shows $f_{\mathrm{e}} / f$ values as a function of silica concentration. The filled circles are from the present work and the empty circles denote the results of Galanti and Sperling [10]. The filled points are determined by averaging $f_{\mathrm{e}} / f$ values of a sample for the three different extension ratios. The error bar corresponds to the standard deviation in the data. AFM analysis of the filled polymers indicated that aggregation of fillers increased significantly with filler content as may be seen from panel $\mathrm{c}$ of Fig. 2. For this reason, filler amounts larger than 5\% were not considered. Comparison of our data with that of Galanti and Sperling shows that the contribution to the energetic component of the elastic force $f_{\mathrm{e}} / f$ and the rate of increase of $f_{\mathrm{e}} / f$ are both larger in our measurements. It is also found that $f_{\mathrm{e}} / f$ values are approximately independent of extension ratios (not shown as a separate figure).

Values of $\mathrm{d} \ln \left\langle r^{2}\right\rangle_{0} / \mathrm{d} T$, obtained by substituting the measured forces and extension ratios in Eq. (4) are displayed in Fig. 6 as a function of silica concentration. Fig. 7 shows force-temperature data for $3 \%$ silica filled PDMS networks in elongation at constant length. Change in force as a function of temperature can be explained by the interplay of two mechanisms: at the molecular level, chains tend to retract with increase in temperature, whereas, the network as a bulk body expands by the usual thermal expansion. Retraction of the chains triggers inducing force, however, expansion of the network relaxes the chains. To

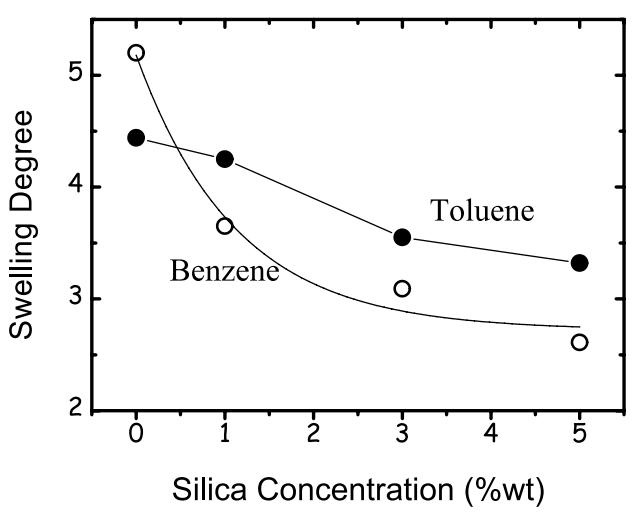

Fig. 3. Equilibrium swelling degree of PDMS networks for unfilled and filled with $1 \%, 3 \%, 5 \%$ wt fumed silica in benzene and toluene.

compensate this effect, extension ratios higher than 1.4 were employed to determine thermoelasticity measurements in our experiments. At moderate elongation, $(\alpha=1.37)$, the latter effect dominates the former. At the higher extension ratios $(\alpha=1.53$ and $\alpha=1.75)$, the rate of change in force increases with respect to temperature at constant elongation.

Elastic moduli of the samples obtained by mechanical experiments and by swelling measurements are presented as a function of filler content in Fig. 8. Empty circles are from stress-strain measurements. The filled circles and open squares are from swelling experiments in toluene and benzene, respectively. The calculations leading to the points in the figure are described in Appendix A. The empty circles are obtained from the initial slopes of stress-strain curves of the samples and indicate the small deformation moduli. The modulus increases linearly with increasing silica concentration. Results from swelling in toluene show approximate agreement with the mechanical data. Swelling in benzene yields larger values in higher silica concentration samples. The difference between results for toluene and benzene may be due to differences between the polymer-solvent interaction parameters for the two systems. However, we cannot at present explain this difference quantitatively using statistical mechanical arguments. At the temperature of measurements, the polymer-solvent interaction parameter data given in Appendix B shows that toluene is a slightly better solvent for PDMS than benzene. This results in smaller degrees of swelling of the same network in benzene than in toluene, and therefore, leads to higher values of the modulus for the benzene-PDMS system.

Table 2

Swelling measurements on end-linked PDMS

\begin{tabular}{|c|c|c|c|c|c|c|}
\hline \multirow{2}{*}{$\begin{array}{l}\text { Silica conc. } \\
\text { Weight } \%\end{array}$} & \multicolumn{3}{|c|}{$E \times 10^{6} \mathrm{~m}^{-1} \mathrm{~kg}^{-2}$} & \multicolumn{3}{|c|}{$M_{\mathrm{c}} \times 10^{3} \mathrm{~g}$} \\
\hline & Toluene & Benzene & Mechanical & Toluene & Benzene & Mechanical \\
\hline Unfilled & 0.26 & 0.13 & 0.19 & 28.4 & 58.4 & 39.4 \\
\hline 0.48 & 0.30 & 0.39 & 0.28 & 25.3 & 19.1 & 26.8 \\
\hline 1.45 & 0.50 & 0.71 & 0.40 & 15.1 & 10.5 & 18.8 \\
\hline 2.45 & 0.62 & 1.36 & 0.62 & 12.2 & 5.5 & 12.1 \\
\hline
\end{tabular}




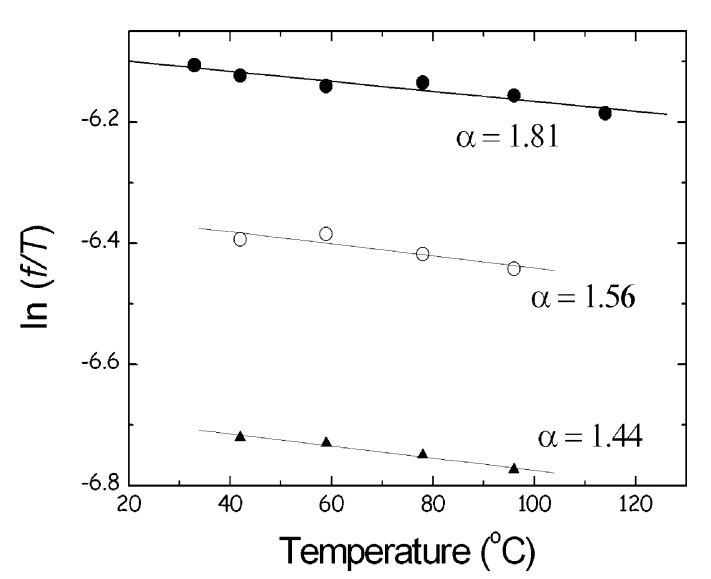

Fig. 4. Thermoelasticity plot of end-linked PDMS with 5\% wt silica. The equations of the best-fitting lines are: $\ln (f / T)=-8.7 \times 10^{-4} T-6.1(\alpha=1.81), \ln (f / T)=-7.0 \times 10^{-4} T-6.3$ $(\alpha=1.56)$ and $\ln (f / T)=-8.3 \times 10^{-4} T-6.7(\alpha=1.44)$. The regression values of the best fitting lines are $0.85,0.82$ and 0.97 , respectively.

\section{Discussion}

The temperature coefficient of PDMS chains are positive with an experimental value of $f_{\mathrm{e}} / f=0.2$ and $\mathrm{d} \ln \left\langle r^{2}\right\rangle / \mathrm{d} T=$ $0.67 \times 10^{-3}[17,4]$. The present results which agree with those of Galanti and Sperling are lower, lying in the range $f_{\mathrm{e}} /$ $f=0.11-0.15$ and $\mathrm{d} \ln \left\langle r^{2}\right\rangle / \mathrm{d} T=0.32-0.45 \times 10^{-3}$.

The observed increase in $f_{\mathrm{e}} / f$ and $\mathrm{d} \ln \left\langle r^{2}\right\rangle / \mathrm{d} T$ with filler content results from the presence of the filler, or more specifically from the filler polymer interface. As suggested by the recent NMR, DSC and AFM measurements, the presence of a mobility gradient of the chains plays important role in the observed temperature dependence $[6,8]$. The chains in a bulk amorphous elastomer have the full freedom of taking all possible configurations, subject to the constraints imposed by the cross-links at their two ends only. However, the presence of nanometer size fillers results in adsorption and immobilization of a significant fraction of these chains on the walls of the filler.

As shown in Appendix B, a path from the surface of one filler to the neighboring one contains about 3-7 chains for

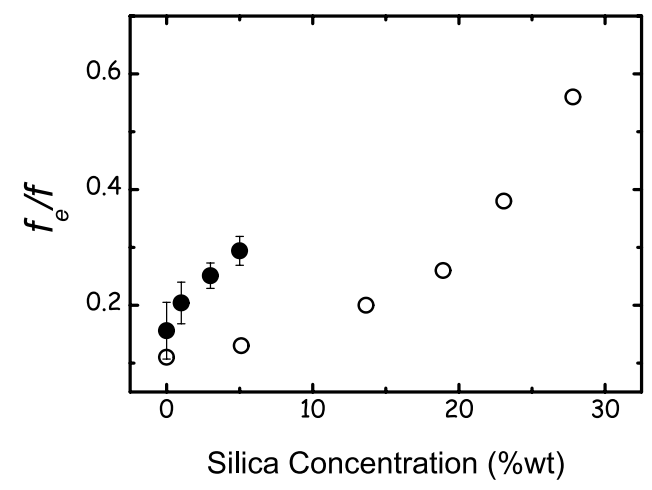

Fig. 5. $f_{\mathrm{e}} / f$ values as a function of silica concentration. Open circles represent results from Ref. [10] and close circles show our experimental data.

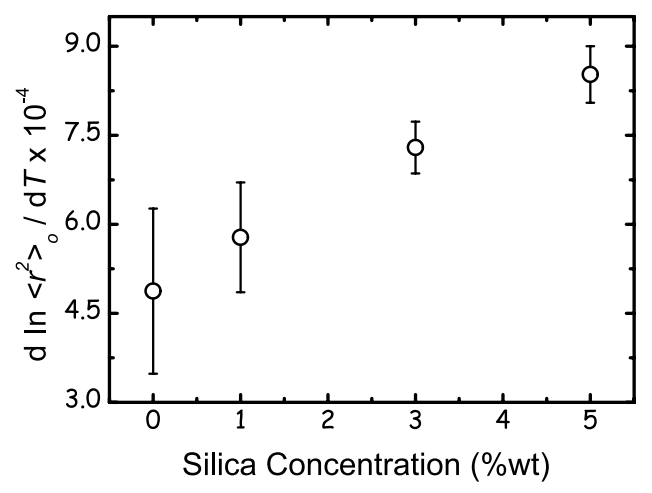

Fig. 6. $\ln \left\langle r^{2}\right\rangle_{0} / \mathrm{d} T$ of PDMS chains as a function of silica concentration.

filler loading of $1 \mathrm{wt} \%$. A fraction of the segments of these chains along the path will be immobilized, due to the presence of the filler, and the basic postulate of an amorphous network chain will hold only for the remaining mobile segments. Uniaxial tensile deformation of the network is expected to move chain segments from the immobilized region, as a result of which, the number of segments in the mobile region will increase. We show in Appendix $\mathrm{C}$ that only a very small number of segments moving from the immobilized to the mobile regions will contribute significantly to the temperature coefficient of the filled networks. The magnitude of this contribution from the interface to the temperature coefficient is significant, and we think that a better understanding of the thermoelasticity of filled elastomers is only possible by a complete understanding of the rubber filler interface.

We would like to add in conclusion that two important factors that affect thermoelasticity of filled networks should be taken into consideration in future work. The first is the effect of precursor chain length. The present study only addresses the effect of fillers for networks with single $M_{\mathrm{c}}$ values for the precursor chains. Thermoelastic effects for networks of different length precursor chains prepared by different filler amounts are expected to be important and should be studied in detail. Secondly, the effect of filler

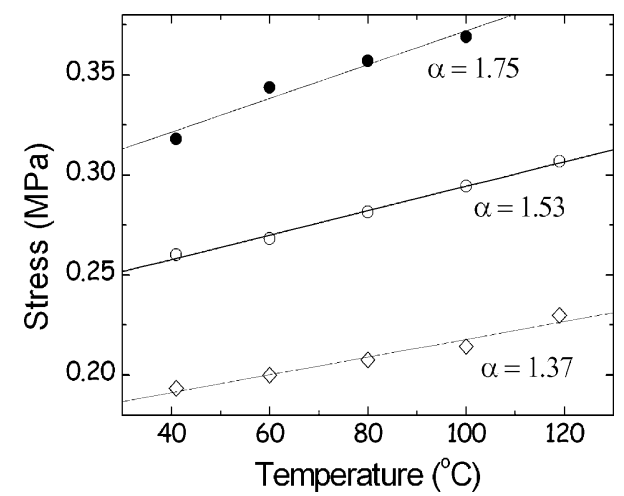

Fig. 7. Typical force-temperature curves at various elongations at constant length. The equations of the best-fitting lines are: $\sigma=8.4 \times 10^{-4} T+0.29$, $\sigma=6.1 \times 10^{-4} T+0.23$, and $\sigma=4.4 \times 10^{-4} T+0.23$. Slopes of the lines decrease with decreasing the extension ratio. 


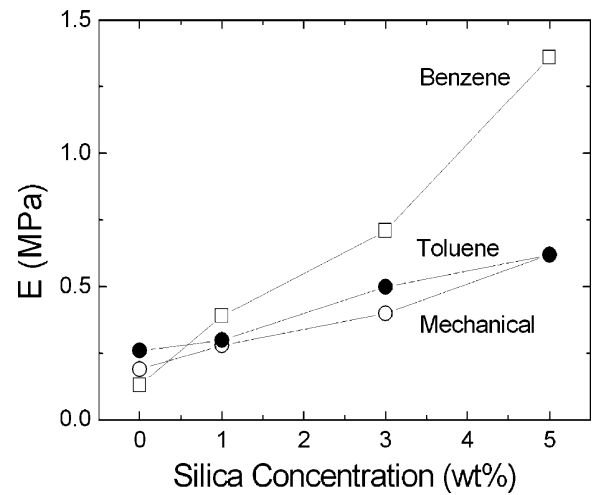

Fig. 8. Elastic modulus of the unfilled and silica filled PDMS networks obtained by mechanical and by swelling in toluene and benzene.

aggregation on thermoelasticity of networks should be studied in more detail. A significant amount of aggregation is observed in the samples of the present study in going from 1 to $5 \%$ silica content, as can be seen from Fig. 2. Aggregation decreases the effective surface area of fillers, and increases the space between neighboring aggregates. These two factors are expected to decrease the filler effect on rubber elasticity, the extent of which could possibly be studied with elastomers having in situ generated fillers.

\section{Acknowledgements}

Thank to Assoc. Prof Canan Baysal for her helpful discussion and suggestions, to Mr Fikri Alemdaroglu for his help at the initial stages of the experiments, and to $\mathrm{Mr}$ Karahan Bulut for his help on the computer systems.

\section{Appendix A. Calculation of degree of end-linking and modulus from swelling data}

The elastic equation of state for an affine network is

$$
\sigma=\frac{\rho R T}{M_{\mathrm{c}}}\left(\alpha-\alpha^{-2}\right)
$$

where $\sigma$ is the stress, $\rho$ is the density of dry chains, taken as $0.97 \mathrm{~g} / \mathrm{cm}^{3}, R$ is the gas constant, $T$ the absolute temperature, taken as $300 \mathrm{~K}$ in the present work, $M_{\mathrm{c}}$ is the molecular weight of chains between two cross-links, and $\alpha$ is the extension ratio. The affine network assumption is plausible due to strict constraints imposed on the chains from the rigid filler particles. Alternatively, the phantom network model could be used where the front factor in Eq. (A1) should be reduced by a factor of 2 . It should be noted that the phantom network model is strictly valid for unfilled swollen networks in which the fluctuations of junctions take place in the large volume obtained as a result of swelling. However, this is not the case for the filled samples used in this study, where fluctuations are supressed due to the presence of fillers. We therefore adopt the affine network model, which is more suitable for the filled networks, swollen or unswollen. For small deformations, Eq. (A1) takes the form $\sigma=3 E \varepsilon$, where $\varepsilon$ is the strain, and the modulus of elasticity is related to $M_{\mathrm{c}}$ as

$E=3 \frac{\rho R T}{M_{\mathrm{c}}}$

Alternatively, $M_{\mathrm{c}}$ may be obtained by swelling from the expression [4]

$M_{\mathrm{c}}=-\frac{\rho V_{1} v_{2 c}^{2 / 3} v_{2}^{1 / 3}}{\ln \left(1-v_{2}\right)+\chi v_{2}^{2}+v_{2}}$

where $v_{2}$ is the volume fraction of polymer, and $\chi$ is the polymer-solvent interaction parameter. $v_{2 c}$ is the volume fraction of polymer during the formation of the polymer, which was not constant throughout the cross-linking stage in the present experiments. An average value of 0.5 is estimated for $v_{2 c}$ which is used in Eq. (A3) for the evaluation of $M_{\mathrm{c}}$. The network is assumed to obey the affine model because the chains are expected to be highly constrained due to the presence of the rigid filler particles. Cross-linking in solution is known to favor loop formation and dangling chains, which could suitably be estimated, but not undertaken in the present work, by spectroscopic or titration methods. However, corrections for these factors would be counterbalanced by the uncertainties resulting from the indeterminate $v_{2 c}$ values that obtain during crosslinking.

In Eq. (A3), the concentration dependence of the $\chi$ parameter is important. For toluene at $25^{\circ} \mathrm{C}$ it is taken as [18]

$\chi=0.48+0.38 v_{2}$

for toluene-PDMS, and as

$\chi=0.49+0.36 v_{2}$

for benzene-PDMS [19].

\section{Appendix B. Calculation of the average size of a path between two neighboring particles}

For the case of well dispersed uniform sized fillers in an elastomer, the average volume fraction $v$ of fillers may be approximated by assuming that the filler particles are arranged in perfect order in a cubic lattice as

$v=\frac{K \pi D^{3}}{6 l^{3}}$

Here, $D$ is the diameter of the fillers, assumed spherical, and $l$ is the distance between two neighboring filler particles. $K$ is a coefficient that depends on the type of arrangement in the lattice. For simple and body centered cubic arrangements, $K=1$ and 2 , respectively. Eq. (B1) rearranges to 
$\frac{l}{D}=\left(\frac{K \pi}{6}\right)^{1 / 3} v^{-1 / 3}$

According to this expression, the role of the indefinite parameter $K$ is inconsequential because of the cube root. For $K=2$, and for weight fractions ranging between 1 and $2 \%$, the $l / \mathrm{D}$ ratio varies between $3.45<l / D>5.93$. For the networks used in the present experiments, using $\left\langle r^{2}\right\rangle_{0}=C n l^{2}$, with $C=6.0, n=675$ and $l=0.167 \mathrm{~nm}$, the root meansquared end to end distance is calculated as $\left\langle r^{2}\right\rangle_{0}^{1 / 2}=10.6 \mathrm{~nm}$. For a particle of $D=15 \mathrm{~nm}, 51<l<89$, corresponding to 3-7 chains between two neighboring particles.

\section{Appendix C. Contributions to the temperature coefficient of PDMS chains}

Consider a chain with $n_{0}$ bonds. Assume that the chain spans the distance between two filler particles, and that it is divided into three parts with $n^{\prime}, n$, and $n^{\prime \prime}$ bonds. Assume that the parts containing the $n^{\prime}$ and $n^{\prime \prime}$ bonds are immobilized at the walls of the two filler particles, and that the middle portion with $n$ bonds can take all possible configurations subject to the connectivity of the chain and the constraints imposed by the two ends. Also assume that bonds are transferred from the immobilized portions containing the $n^{\prime}$ and $n^{\prime \prime}$ bonds as the temperature is increased. The mean-squared dimensions of the central portion containing the $n$ segments will change according to

$\frac{\mathrm{d} \ln \left\langle r^{2}\right\rangle}{\mathrm{d} T}=\left(\frac{\partial \ln \left\langle r^{2}\right\rangle}{\partial T}\right)_{n}+\left(\frac{\partial \ln \left\langle r^{2}\right\rangle}{\partial n}\right)_{T} \frac{\mathrm{d} n}{\mathrm{~d} T}$

The first term on the right-hand side of Eq. (C1) is the well known temperature coefficient expression, and the second term reflects changes in dimensions when bonds are released from the immobilized ends into the mobile part of the chain when the temperature is increased. For chains for which $\left\langle r^{2}\right\rangle=C_{\infty} n l^{2}$, Eq. (C1) reduces to

$\frac{\mathrm{d} \ln \left\langle r^{2}\right\rangle}{\mathrm{d} T}=\frac{\mathrm{d} C_{\infty}}{\mathrm{d} T}+\frac{1}{n} \frac{\mathrm{d} n}{\mathrm{~d} T}$

The Rotational Isomeric States (RIS) calculations have been performed for PDMS chains from which the limiting value of $0.75 \times 10^{-3}$ have been obtained for $\mathrm{d} C_{\infty} / \mathrm{d} T$ [17]. The second term, $(1 / n) \mathrm{d} n / \mathrm{d} T$, consists of two parts, the first part varies with inverse of the number of repeat units. The second part equates to the number of bonds moving from the immobilized to the mobile section of the chain per degree increase in the temperature. RIS calculations indicate that $C_{\infty}$ is reached for PDMS chain when $n=200$. Thus, if only one bond moves from each end to the mobile part of the chain per 10 degrees increase in temperature, the contribution of the second term of Eq. (C2) will equate to $1.0 \times$ $10^{-3}$, which is already larger than the first term. It should be noted that these calculations are only approximate and are only estimating the order of magnitude of the two different contributions. If the network chains are not long enough, then the first term in Eq. (C2) reads $\mathrm{d} C / \mathrm{d} T$, and may depend on the number of bonds. We have performed RIS calculations for different length chains, with the energy and geometry parameters given in Ref. [20]. For $n=20, \mathrm{dCl}$ $\mathrm{d} T=0.50 \times 10^{-3}$, and converges rapidly to the limiting value of $0.75 \times 10^{-3}$, indicating that this term does not depend significantly on chain length.

\section{References}

[1] Osman MA, Atallah A, Müller M, Suter UW. Polymer 2001;42:6545.

[2] Yuan WQ, Mark JE. Macromol Chem Phys 1999;200:206.

[3] Breiner JM, Mark JE. Polymer 1998;39(22):5483.

[4] Mark JE, Erman B. Rubberlike elasticity a molecular primer. New York: Wiley Interscience; 1988, p. 61.

[5] Erman B, Mark JE. Structures and properties of rubberlike networks. Oxford: Oxford University Press; 1997.

[6] Litvinov WM, Barthel H, Weis J. Macromolecules 2002;35:4356.

[7] Gussoni M, Greco F, Mapelli M, Vezzoli A, Ranucci E, Ferruti P, et al. Macromolecules 2002;35:1722.

[8] Berriot J, Lequeux F, Monnerie L, Montes H, Long D, Sotta P. J NonCryst Solids 2002;307-310:719.

[9] Cosgrove T, Roberts C, Garasanin T, Schmidt GR, Gordon VG. Langmuir 2002;18:10080.

[10] Galanti AV, Sperling LH. Polym Eng Sci 1970;10(3):177.

[11] Shackelford JF. Introduction to materials science for engineers. 5th ed. New Jersey: Prentice-Hall Inc; 2000, p. 802 and 808.

[12] Brandrup J, Immergut EH, Grulke EA. Polymer handbook. 4th ed. 199 V-168.

[13] Ciferri A, Hoeve CA, Flory PJ. J Am Chem Soc 1961;83:1015.

[14] Flory PJ, Hoeve CA, Ciferri A. J Polym Sci 1959;34:337.

[15] Flory PJ, Ciferri A, Hoeve CA. J Polym Sci 1960;45:235.

[16] Flory PJ. Statistical mechanics of chain molecules. New York: Interscience; 1969, p. 39.

[17] Mark JE. Macromol Rev 1976;11:135.

[18] Schneider A, Schuld N, Bercea M, Wolf BA. J Polym Sci, Part B: Poly Phys 2004;42(9):1601.

[19] Flory PJ, Shih H. Macromolecules 1972;5:761.

[20] Flory PJ, Crescenzi V, Mark JE. J Am Chem Soc 1964;86:146. 